\title{
Pulling T cells to pathogens
}

Vaccines work because they train memory $T$ and $B$ cells to act as armed sentinels that guard against future attack by a pathogen. But to effectively block infection, these sentinels must be stationed at the portal through which the pathogen enters. A recent Nature paper by Shin and Iwasaki ${ }^{1}$ describes a new vaccination protocol that boosts the numbers of $T$ cells at a mucosal site of pathogen invasion. Through local application of chemokines in mice, the authors 'pull' effector $T$ cells made in response to a vaccine against herpes simplex virus 2 (HSV-2) from the blood into the vaginal cavity, reducing viral titers in surrounding dorsal root ganglia.

After entering the vaginal cavity, HSV-2the causative agent of genital herpesreplicates in mucosal epithelial cells before spreading to innervating neurons and establishing latency within the dorsal root ganglia. HSV-2 periodically reawakens from dormancy and infects and kills mucosal epithelial cells. These reactivations cause the characteristic lesions of clinical genital herpes.

The strategy developed by Shin and Iwasaki ${ }^{1}$ exploits the normal defensive process triggered by HSV-2 infection whereby $T$ cells are attracted to infected mucosa in response to virus-induced inflammation. In mice, HSV-2 infection causes $\mathrm{CD}^{+}{ }^{+} \mathrm{T}$ cell production of interferon- $\gamma$, which elicits production of the chemokines CXCL9 and $\mathrm{CXCL10}$, which in turn recruit effector $\mathrm{CD}^{+} \mathrm{T}$ cells expressing CXCR3, the receptor for CXCL9 and CXCL10. The authors reasoned that mimicking just one step of this inflammatory cascade-production of CXCL9 and CXCL10 - might pull in protective $T$ cells while bypassing unwanted vaginal inflammation.

The authors immunized mice subcutaneously with an attenuated strain of HSV-2 and, 5 days later, injected a solution of CXCL9 and CXCL10, or saline, into the vagina. Chemokine application increased the number of $\mathrm{HSV}-2$-specific $\mathrm{CD}^{+} \mathrm{T}$ cells-but, importantly, not other inflammatory cells - in the vaginal mucosa. The $\mathrm{CD}^{+} \mathrm{T}$ cells remained in the vaginal cavity

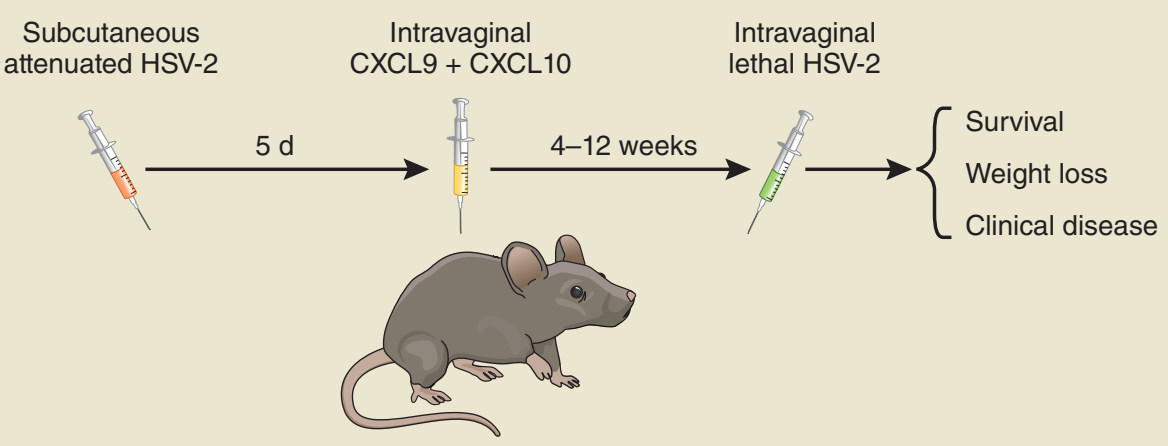

for at least 12 weeks. Compared with mice receiving only subcutaneous immunization (the 'prime' strategy), those receiving subcutaneous immunization followed by intravaginal chemokine injection (the 'prime and pull' strategy) lost less weight, showed less severe clinical disease and survived when infected 4-12 weeks later with a lethal dose of HSV-2. HSV-2 viral titers, although not significantly reduced in vaginal secretions, were markedly lower in dorsal root ganglia, suggesting that the protective memory $T$ cells recruited to the vaginal mucosa may act by preventing HSV-2 spread from epithelial cells to neurons.

Although the approach shows promise in this mouse model, more work is needed to determine if it can safely prevent HSV-2 infection and/or reactivation in humans. "Whether preparations of CXCL9 and CXCL10 would be safe and elicit a response of similar specificity and potency after vaginal administration in humans is unclear," says Larry Corey of the Fred Hutchinson Cancer Research Center in Seattle. Also uncertain is how long the protective benefits of prime-and-pull vaccination would last. "In this mouse model, pathogen challenge is done at most 12 weeks after vaccination. Whether protection would be observed at clinically relevant later time points is not clear," says Louis Picker of Oregon Health \& Science University in Portland, adding that "there are unfortunately currently no good large-animal models for looking at herpes virus reactivation from latency."

The authors suggest that their vaccination strategy might improve protection against other sexually transmitted infections, including HIV. However, because the vaccine seemed to work by preventing dorsal root ganglia infection rather than viral replication in the vaginal mucosa, the approach may not be well-suited to non-neurotropic viruses that proliferate rapidly upon mucosal infection. "For HIV or SIV infections to be stopped by 'pulled' $T$ cells in portals of entry, local virus replication would have to be effectively abrogated before lymphatic or hematogenous spread, likely requiring a very high frequency of virus-specific effector cells," says Picker.

But even if the efficacy of pulled T cells proves too low in some situations, this general concept may be applicable to other kinds of immune cells. "It may be intriguing to try to use this general prime-and-pull strategy to recruit circulating effector B cells into the effector site because it's currently hard to elicit local B-cell responses," says Picker, adding that "these effector $B$ cells might form plasma cells, which could secrete antibodies at the local site of infection." With chemokines that attract the right B-cell populations, this strategy might even be useful for HSV-2. As Corey points out, "the majority of human data, especially those that indicate that passive transfer of specific antibodies from mother to infant reduces the risk of transmission, indicates antibodies likely play a significant role in reducing acquisition of infection."

\section{Christine Borowski, Senior Editor,} Nature Biotechnology

1. Shin, H. \& Iwasaki, A. Nature advance online publication, doi:10.1038/nature11522 (2012). 